\title{
Geographic Differentiation and Population Genetic Structure of Moniliophthora roreri in the Principal Cocoa Production Areas in Colombia
}

Yeirme Y. Jaimes, Corpoica, Research Center La Suiza, Rionegro-Santander, Colombia; CAPES PEC-PG Fellow; and Plant Protection Department, Faculty of Agricultural Sciences, São Paulo State University, Botucatu, SP, Brasil; Carolina Gonzalez, Corpoica, Research Center Tibaitata, Mosquera, Colombia; Jairo Rojas, Corpoica, Research Center La Suiza; Omar E. Cornejo, School of Biological Sciences, Washington State University, Pullman, WA; Maria F. Mideros and Silvia Restrepo, Department of Biological Sciences, Universidad de los Andes, Bogotá, Colombia; Christian Cilas, CIRAD, UR Bioagresseurs, Campus de Baillarguet, TA A-106/D, 34398 Montpellier Cedex 5, France; and E. L. Furtado, CNPq Fellow, Plant Protection Department, Faculty of Agricultural Sciences, São Paulo State University, Botucatu, SP, Brasil

\begin{abstract}
Jaimes, Y. Y., Gonzalez, C., Rojas, J., Cornejo, O. E., Mideros, M. F., Restrepo, S., Cilas, C., and Furtado, E. L. 2016. Geographic differentiation and population genetic structure of Moniliophthora roreri in the principal cocoa production areas in Colombia. Plant Dis. 100:1548-1558.

Frosty pod rot (FPR) disease on cocoa, caused by Moniliophthora roreri, is one of the most devastating cocoa disease in the Western Hemisphere. In Colombia, the disease is particularly severe in the Magdalena Valley, which is considered the possible center of origin for the pathogen species. We analyzed the genetic diversity of isolates from the departments of Santander, Antioquia, Tolima, and Huila in Colombia using 23 simplesequence repeats (SSR) markers. In total, 117 different multilocus genotypes were found among 120 isolates, each one representing a unique haplotype. High mutation rates in the SSR and gene flow can explain the high levels of diversity. Also, the observed and standardized indexes of association $\left(I_{A}\right.$ and $\left.\check{r} d\right)$ indicate that the populations of $M$. roreri are clonal. Furthermore, given the high haplotype diversity and the significant

linkage disequilibrium observed, we hypothesize that $M$. roreri could be a primarily asexual species undergoing sporadic recombination or partial recombination through parasexuality. A Bayesian clustering analysis implemented by STRUCTURE showed that the most probable number of genetic groups in the data was three, confirming the geographical differentiation among isolates. Similar results were obtained by a discriminant analysis of principal components, a principal coordinate analysis, and a neighbor-joining tree from microsatellite loci base on Nei distance. Cacao genotypes and environmental variables did contribute to the genetic differentiation of the groups. We discuss how this information could be used to improve the management of FPR at the regional level.
\end{abstract}

Moniliophthora roreri (Cif.) H. C. Evans, Stalpers, Samson \& Benny, causal agent of frosty pod rot (FPR), is a parasitic fungus of plants belonging to the Marasmiaceae family. For many years, $M$. roreri was considered the anamorph of an ascomycete. Taxonomic and molecular techniques clarified its position as a basidiomycete and demonstrated its close genetic relationship to the witches' broom pathogen, M. perniciosa (Bailey et al. 2013). FPR disease occurs in the major cacao-producing areas in the Western hemisphere. $M$. roreri, a highly specialized fungus, attacks only fruit of the species of the close genera Herrania and Theobroma, causing internal and external pod damage that results in total pod loss. The presence of the disease in the cacao tree (Theobroma cacao) has had such devastating effects on yield that long-term economic viability of the crops has been compromised (Phillips-Mora et al. 2007).

At this moment, the feasible strategies for managing FPR are better agronomic practices combined with improved planting and biological control methods, under an integrated management approach (Ali et al. 2015). To date, there are not cacao clones immune to $M$. roreri, and the clones with higher tolerance developed by the Centro Agronómico Tropical de Investigación y Enseñanza in Costa Rica have slowly decreased in tolerance after 15 years. These observations raise questions about the possibility that the pathogen may be in the process of overcoming or breaking tolerance (Ali et al. 2015). A

Corresponding author: C. Cilas; E-mail: christian.cilas@ cirad.fr

*The $\boldsymbol{e}$-Xtra logo stands for "electronic extra" and indicates that two supplementary tables are published online.

Accepted for publication 30 March 2016.

http://dx.doi.org/10.1094/PDIS-12-15-1498-RE

(C) 2016 The American Phytopathological Society previous study has characterized the differential expression of genes during the successful infections when controlled infections on tolerant and susceptible clones infections are compared (Bailey et al. 2014). Some of these differentially expressed genes are involved in changes in metabolism and the induction of fungal stress responses putatively designed to mitigate plant defense mechanisms (Bailey et al. 2014). In general, $M$. roreri is a highly adaptable fungus, able to thrive under a wide range of environmental conditions. It can be found from sea level to over $1,000 \mathrm{~m}$ above sea level and in microclimates that range from very dry to very humid (Evans 1981; Phillips-Mora and Wilkinson 2007). This level of adaptation to different environments, and the huge number of long-lived spores that are generated by each infection has made $M$. roreri a very effective pathogen and a formidable invader of new geographical regions (PhillipsMora and Wilkinson 2007).

It is a general understanding that increased genetic variation in fungi populations would allow these pathogens to readily adapt to changing environment conditions and breeder or natural selective pressures, such as hosts with different degrees of tolerance or resistance to the infections (Bock et al. 2014; McDonald 1997; Thrall et al. 2002). Therefore, a proper understanding of $M$. roreri genetic variation is fundamental to establish a breeding program based in an effective and sustainable resistance (Ali et al. 2015). One of the main limitations in the study of nonmodel organisms such as $M$. roreri is the lack of species-specific markers such as simple-sequence repeats (SSR) or single-nucleotide polymorphisms (SNP), which are considered robust for detailed genotypic assessment of fungi and have become commonly used to assess genetic diversity (Dutech et al. 2007; Frenkel et al. 2012). These markers are highly reproducible and they allow genotyping directly with DNA extracted from diseased plant tissue. Of the two, the SSR markers may be a more economical and rapid method for genetic diversity studies (Jia et al. 2015), especially in countries with limited access to resources for the widespread development of highthroughput SNP detection technologies. 
The development of genetic resources for the study of $M$. roreri, although limited, has provided the possibility to start examining some of these questions. Previous studies preformed using amplified fragment length polymorphisms (AFLP) and intersimple sequence repeat (ISSR) analyses have shown that $M$. roreri has five genetic groups (Phillips-Mora et al. 2007). The groups Co-East, Co-Central, and Bolivar apparently are centered in the northeast of Colombia. Of these, the Bolivar group presents the widest distribution, which includes Venezuela and Peru. Another group, the Co-West, also presents a wide geographic distribution, and can be found in the northwest of Colombia, Central America, and Ecuador. Finally, the group Gireli is restricted to northern Ecuador (Phillips-Mora et al. 2007).

In contrast to the five groups reported by Phillips-Mora et al. (2007), SNP marker analysis showed that M. roreri isolates could be grouped into two major clusters (Ali et al. 2015). Group b has wider geographic range and higher genetic differentiation between locations and lower local genetic diversity. Group A, which is confined to Colombia, has a significant diversity resulting in one groups with four subgroups. The geographic range of subgroup I is centered on the Upper Magdalena Valley, crosses the Central Cordillera of the Andes through the north side, then moves to the south by the Cauca Valley. Similarly, the geographic range of subgroups II and IV runs from the east of Upper Magdalena Valley to the west along to the Pacific Coast down to Ecuador. Subgroup III is located toward the Eastern Cordillera of the Andes. It has been observed that isolates from group $b$ have dispersed from the north of the Upper Magdalena Valley to the west, and few isolates were able to reach and move to the south along the coast (Ali et al. 2015).

In the Phillips-Mora et al. (2007) study, 12 M. roreri isolates restricted to Santander and the central region of Colombia were grouped in two genetic groups. On the other hand, Ali et al. (2015) showed a major diversity of the pathogen in terms of distribution within and dispersion from Santander regions to other areas. Based on these results, this region is considered to be the possible center of origin for $M$. roreri. In addition, the clonal state of $M$. roreri was reported, suggesting a dispersion of the pathogen clones from the Upper Magdalena Valley through time and subsequent localized genetic drift associated with these unique isolates and events. This result was obtained in the absence of heterozygosity for SNP markers within Colombia, suggesting that $M$. roreri isolates do not reproduce by sexual recombination very often (Ali et al. 2015).

The number of isolates employed in the studies aforementioned has been rather limited, making it difficult to unravel the geographic structures and pattern of dispersion of fungus in Colombia and the rest of the region. In order to obtain a clearer picture of the genetic and geographic pattern of differentiation within Colombia, more isolates, especially from the Upper Magdalena Valley, should be analyzed. Using the recently published sequenced genome of $M$. roreri (Meinhardt et al. 2014), we developed 23 specific SSR markers for the analysis of isolates of the Co-Central, Co-East, and Co-West genetic groups reported by Phillips-Mora et al. (2007). The rationale for focusing our analysis on isolates belonging to these groups is based on the observations that the Co-East and Co-Central groups are the most variable and endemic in South America, suggesting that the center of genetic diversity and, potentially, geographic origin of the species is located in Colombia (Phillips-Mora et al. 2007). Our analysis of 120 isolates of $M$. roreri obtained from the four main cocoa-producing departments of Colombia allowed us to identify three main genetic groups using a Bayesian clustering algorithm. We discuss the implications of these results in terms of the potential for adaptation and management of the disease caused by this pathogen of enormous economic importance for the region.

\section{Materials and Methods}

Fungal material and DNA extraction. From 2013 to 2014, pods with FPR symptoms were collected from four main cocoa-producing departments of Colombia from susceptible trees (ICS 39, ICS 60, or ICS 1) and from tolerant trees (ICS 95) in farmers' fields. Five farmers' fields were selected in each locality using, as criteria, the origin of the available vegetal material and the distance higher than $10 \mathrm{~km}$ between fields. In total, 13 localities were sampled: Apartado, Turbo, and Maceo in Antioquia Department, Campo Alegre, and Rivera in Huila Department; Ataco and Planadas in Tolima Department; and Cimitarra, El Carmen, San Vicente, Lebrija, El Playon, and Rionegro in Santander Department (Fig. 1). From each field, diseased cacao fruit were collected with initial or intermediary symptoms of FPR from trees previously selected. This vegetal material was washed and surface sterilized as described by Arbeláez (2010). The isolates were obtained from fruit fragments of approximately $1 \mathrm{~cm}^{3}$ placed onto Sabouraud $4 \%$ dextrose agar (Merck) supplemented with V8 juice (20\% [wt/vol] V8 juice) at $27 \pm 1^{\circ} \mathrm{C}$. The 120 strains of $M$. roreri obtained were purified in petri dishes on Sabouraud 4\% dextrose agar (Merck) at $27^{\circ} \mathrm{C}$ (Supplementary Table S1).

Morphological characterization of fungal isolates. One mycelium plug ( $5 \mathrm{~mm}$ in diameter) taken from 12-day-old colonies was transferred to the center of a $15-\mathrm{cm}$ petri dish on Sabouraud $4 \%$ dextrose agar (Merck) supplemented with V8 juice $(20 \%$ [wt/vol] V8 juice). The petri dish containing the mycelium plug was sealed and incubated in a photoperiod of $12 \mathrm{~h}$ of light and $12 \mathrm{~h}$ of darkness at $27^{\circ} \mathrm{C}$ for 16 days. Each isolate was replicated four times. The petri dishes were randomly placed into a Precision incubator (Thermo Scientific). Mean diameter of colony was recorded each $24 \mathrm{~h}$ and these data were used to calculate the relative growth rate of the colony. At the 16th day, certain characteristics of the colony were registered (color using the Munsell soil color chart (http://munsell.com/colorproducts/), shape, margin presence or absence of sectors, texture, rings position, and spores per square centimeter), as described by Arbeláez (2010). Finally, length and width of 30 spores per isolate were registered using a Motic digital camera adapted to optical microscope CX41 (Olympus) at $\times 40$. Data were obtained from processed images with the Motic Images Plus software v.2.0. All variables were classified by experimental unit and were submitted to principal component analysis (PCA) in the software InfoStat v.2014.

Microsatellite loci detection and primer design. We identified potential regions with SSR in the M. roreri genome available in GenBank (Meinhardt et al. 2014) under accession number MCA2977, using GMATO (Wang et al. 2013) (default settings). To reduce the large number of potential microsatellite loci detected, sequences containing microsatellites with two nucleotide motifs were discarded as well as those located in regions encoding genes with conserved function. Thereby, 23 SSR with three motifs were selected and the primers were designed with the primer-Blast tool (http://www.ncbi. nlm.nih.gov/tools/primer-blast/index.cgi?LINK_LOC=BlastHome). The major parameters of primer selection were as follow: length of polymerase chain reaction (PCR) products in four different size classes (50 to 120, 120 to 200, 200 to 300, and 300 to 400), length of each primer of 18 to $22 \mathrm{bp}$, melting temperature at 56 to $60^{\circ} \mathrm{C}$, and $\mathrm{G}$ plus $\mathrm{C}$ content in any primer of 45 to $55 \%$. The primers were labeled with 6-FAM, VID, NED, and ROX fluorophore. To establish highthroughput SSR analysis in this study, we placed 23 SSR markers arranged into three PCR multiplexed panels according to their fragment size and fluorescent dye label (Supplementary Table S2).

Microsatellite amplification and genotyping. Genomic DNA was extracted from isolates of $M$. roreri using the ZR Fungal/Bacterial MiniPrep Kit (Zymo Research), according to the manufacturer's instructions. The genomic DNA of $M$. roreri used in this study was extracted and purified from fungal mycelium incubated on Sabouraud $4 \%$ dextrose agar (Merck) for 15 days. For PCR amplification, the reactions were performed in a total volume of $25 \mu \mathrm{l}$ with approximately $50 \mathrm{ng}$ of genomic DNA; $200 \mu \mathrm{M}$ each dNTP; $1 \mu \mathrm{M}$ forward primer labeled with either 6-FAM, VID, NED, or ROX fluorophore; $1 \mu \mathrm{M}$ nonlabeled reverse primer; $1.5 \mathrm{mM} \mathrm{MgCl}_{2}$; and $1 \times$ Buffer Gold and AmpliTaq Gold polymerase (Applied Biosystems).

The PCR was carried out using an icycler Thermal Cycler (Bio$\mathrm{Rad})$ with the following conditions: an initial denaturation at $94^{\circ} \mathrm{C}$ for $10 \mathrm{~min} ; 37$ cycles of denaturation at $92^{\circ} \mathrm{C}$ for $90 \mathrm{~s}$, annealing at $56^{\circ} \mathrm{C}$ for $60 \mathrm{~s}$, with increase of $1^{\circ} \mathrm{C}$ every cycle until reaching $60^{\circ} \mathrm{C}$, and elongation at $72^{\circ} \mathrm{C}$ for $90 \mathrm{~s}$; and ending with an extension at $72^{\circ} \mathrm{C}$ for $52 \mathrm{~min}$. Labeled amplification products were resolved onto an automated 310 ABI PRISM DNA genetic analyzer (Applied 
Biosystems), using performance-optimized polymer 4 as an internal size standard. The PCR fragments were detected with the GeneScan analysis software (v.3.1; Applied Biosystems). Multilocus genotypes (MLG) were determined using the set of 23 nuclear microsatellite loci developed for this study. Analysis was conducted for 120 isolates of $M$. roreri. For each SSR multiplex running, the reproducibility intraand interassay was assessed including the same PCR product in all runs.

Population genetic analysis and structure. For the population genetic analysis, an MLG was assigned to each isolate with the poppr package (Kamvar et al. 2014) implemented in R software v.2.1.0. This $\mathrm{R}$ packages introduces core functions for analysis, including options that consider mixed modes of reproduction (asexual and sexual), typically observed in microbial populations. Isolates with the same MLG were treated as clones using clone-correction implemented in the poppr package in order to eliminate the redundancy in the collection that results from asexual reproduction of the isolates (Chen and McDonald 1996; Kumar et al. 1999). The population genetic structure was estimated for all isolates of $M$. roreri using four levels of stratification: (i) clone, (ii) locality, (iii) department, and (iv) altitude. For each stratum, frequencies within subpopulations at each locus were tested for departures from Hardy-Weinberg equilibrium (HWE) in the poppr package (Kamvar et al. 2014). This test was performed to explore random mating (Michalakis and Excoffier 1996). Genotypic diversity (Nei 1987), evenness, and the ShannonWiener index of diversity and population summary statistic were also determined using the poppr package in R software v.2.1.0. Each statistic was calculated for each data subset with and without corrections for sample size (Chao and Shen 2003). Additionally, we compared the ratio of observed multilocus genotypic diversity to that expected under conditions of sexual reproductions, as described by Stoddart and Taylor (1988).

In order to determine the degree of clonality and the potential for recombination in the population we estimated linkage disequilibrium (LD) across SSR markers using the standardized index of association $\left(I_{A}\right)$, and also with a measure corrected for the number of loci $(\check{r} d)$ (Agapow and Burt 2001). A lack of association between any pair of loci may be interpreted as being due to recombination, whereas linkage or homoplasy might cause deviations from expected genotypic frequencies under random mating in some pairs of loci (Agapow and Burt 2001). For this analysis, we used 1,000 random permutations and missing data were not considered.

We tested for admixture under a model without supervision using Bayesian model based clustering software Structure v.2.3 (Pritchard et al. 2000). We explored clustering under two different models, one allowing for admixture (individuals may have mixed ancestry, which would be consistent with a scenario in which some degree of recombination has occurred historically) and a no-admixture model, allowing correlated allele frequencies (Falush et al. 2003). Searches with ancestral number of cluster ranging from $\mathrm{K}=1$ to 15 were performed in independent runs with 100,000 generations and a burn-in of 5,000. $\mathrm{K}$ was established by taking into account the mean estimated likelihood probability (Pritchard et al. 2000) and the second-order rate of changes in the log probability $(\Delta \mathrm{K})$ (Evanno et al. 2005), implemented in Structure Harvester v.0.6.93 (Earl and vonHoldt 2012).
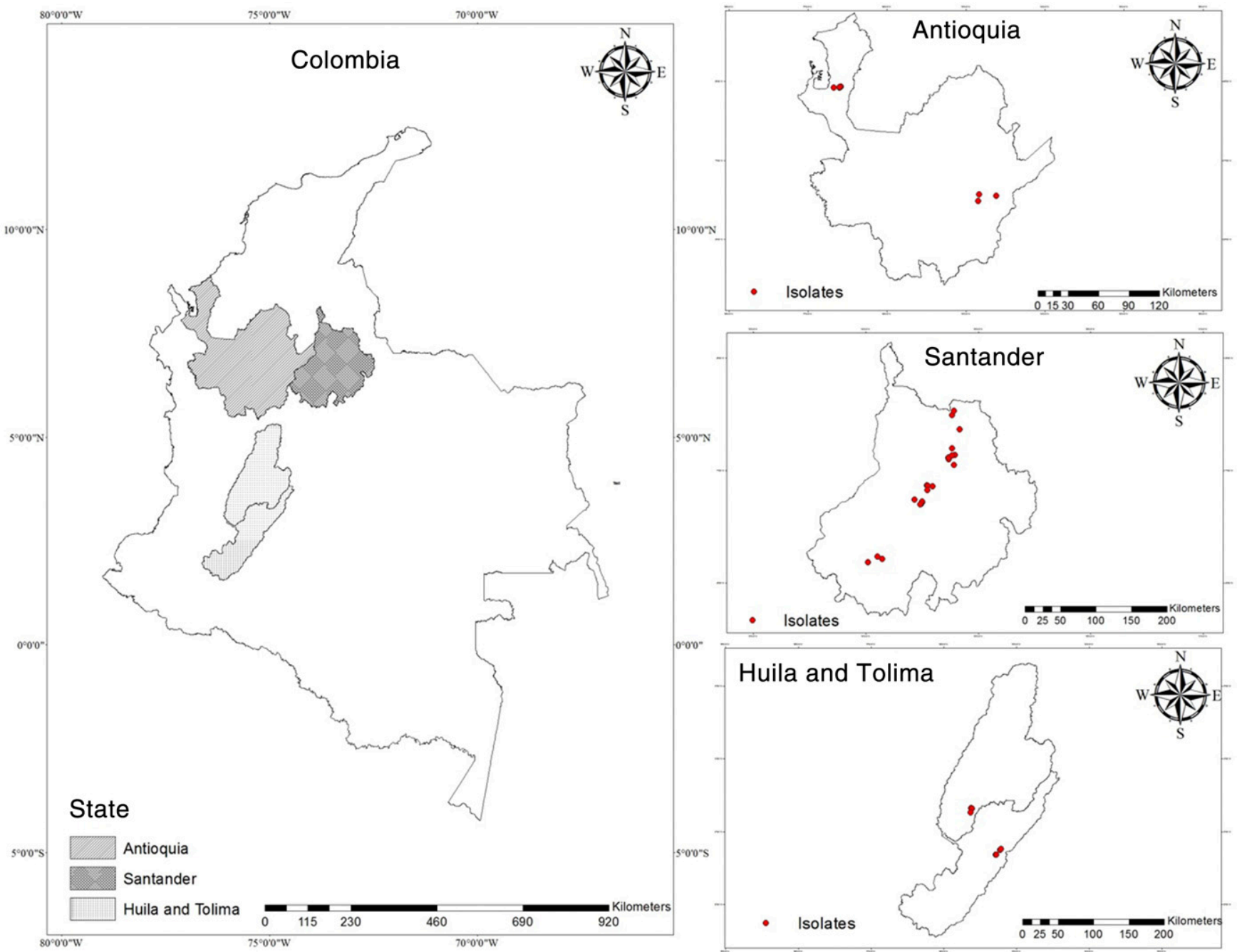

Fig. 1. Geographical distribution of Moniliophthora roreri isolates from four departments in Colombia. Dots indicate the sampling areas. The map was obtained using the ArcGis software v.10.1. 
Population genetic structure was further analyzed by conducting an analysis of molecular variance (AMOVA) using poppr package in $\mathrm{R}$ software v.2.1.0. For this analysis, the department stratum was considered as population and each one of the other strata as subpopulations. It was conducted to partition variance into covariance components due to intraindividual, intersubpopulation, and interpopulation differences. Covariance components were used to calculate $\phi$ value to infer gene flow and population differentiation (Grünwald and Hoheisel 2006). For these analyses, clone-correction was used to remove repeated MLG. Statistical significance was assessed with randomization tests with 999 permutations (Bennett and Stone 2016).

In addition to the structure analysis, we performed a discriminant analysis of principal components (DAPC). This technique extracts information from genetic datasets (multivariate in nature) by first performing a PCA on predefined groups or populations, then using the PCA factors as variables for discriminant analysis, which seeks to maximize the intergroup component of variation (Jombart et al. 2010). DAPC was implemented in the adegenet package for $\mathrm{R}$ (http://adegenet.r-forge.r-project.org/) (Jombart 2008). Level of clustering was assessed with the function find.clusters run over 150 components. We used Akaike Information Criterion and Bayesian Information Criterion to choose the best model, and therefore the number and nature of clusters. Additionally, we also computed a principal coordinate analysis using the adegenet package (Jombart 2008), plotting individuals in the most significant axes space according to their genetic similarities. Genetic divergence among isolates was quantified by calculating the neighbor-joining tree from microsatellite loci with 1,000 bootstrap iterations based on Nei distance. We explored the population structure with a diverse set of relatively orthogonal methods in order to ensure robustness of our results.

\section{Results}

Morphological characteristics. Growth on agar Sabouraud 4\% dextrose Agar (Merck) supplemented with V8 juice (20\% [wt/vol] V8 juice) was slow: colonies attained a diameter from 11 to $90 \mathrm{~mm}$ after 16 days. A feathery margin was a predominant characteristic in all isolates. Most isolates presented rings in the colony but their distribution varied among isolates. Colony colors alternated between whites (7.5 YR 8/1 and 10 YR 8/1), light to dark browns
(7.5 YR 6/4, 10 YR 7/3, 10 YR 5/3, 10 YR 4/3, 7.5 YR 5/2, $10 \mathrm{YR} 4 / 3$, and $10 \mathrm{YR} 3 / 3$ ), light grays (10 YR 7/2 and $10 \mathrm{YR}$ $7 / 1$ ), and light to dark brownish grays (10 YR 6/2 and 10 YR 4/2). Few colonies showed weak or pale red colors (10 R 4/3 and $10 \mathrm{R}$ $6 / 2$ ), presence of sector, and absence of rings in the colony. Spore of all isolates were typically globose to subglobose in shape, with diameters of 6.64 to 9 by 8.64 to $13.28 \mu \mathrm{m}$.

The first and second components of the PCA performed with morphological data accounted for $19.6 \%$ (PC I) and $13.4 \%$ (PC II) of the total variation, respectively. Higher morphological variability was observed among isolates than among groups of isolates sharing the same geographical origin. Indeed, morphological characterization, based on the variables measured in this study, did not allow for a clear discrimination of geographical origin in M. roreri (Fig. 2), suggesting that underlying causes for phenotypic variation did not link to the genetic differentiation between localities (see below).

Genotyping and population statistics using microsatellite loci. From the $M$. roreri genome published in GenBank, we identified 714 trinucleotides, 16 SSR pentanucleotides, and 5 SSR heptanucleotides. After a screening selection, the SSR located in conserved regions and those with less than five motif repeats were discarded. Finally, 23 SSR trinucleotides with five or more motif repeats were selected for marker development. These species-specific microsatellite loci were used to genotype $120 \mathrm{M}$. roreri strains. All of the loci were haploid and most of them were polymorphic in all populations, except for Moni 11 (monomorphic for all isolates). An average of 4.87 alleles/locus were detected over 23 loci ranging from 1 (Moni 11) to 13 (Moni 19) alleles per locus. Both Moni 19 and Moni 21 were the most informative loci, with 13 and 7 alleles, respectively. The locus Moni 19 had the highest Simpson diversity (0.873). Moni 15 had the highest expected heterozygosity (or gene diversity; 0.9) and Moni 23 had the most evenly distributed alleles (0.956). We excluded those samples in the population with a proportion of missing data $>0.05$. After Bonferroni correction for multiples comparison, almost all loci (21 of 23) showed an excess of homozygotes and significant deviation from HWE $(P>0.05)$, suggesting the existence of inbreeding or population subdivision (i.e., Wahlund effect).

In total, 117 MLG were detected for all populations (Table 1). The isolates showed high genetic diversity, with most MLG being represented

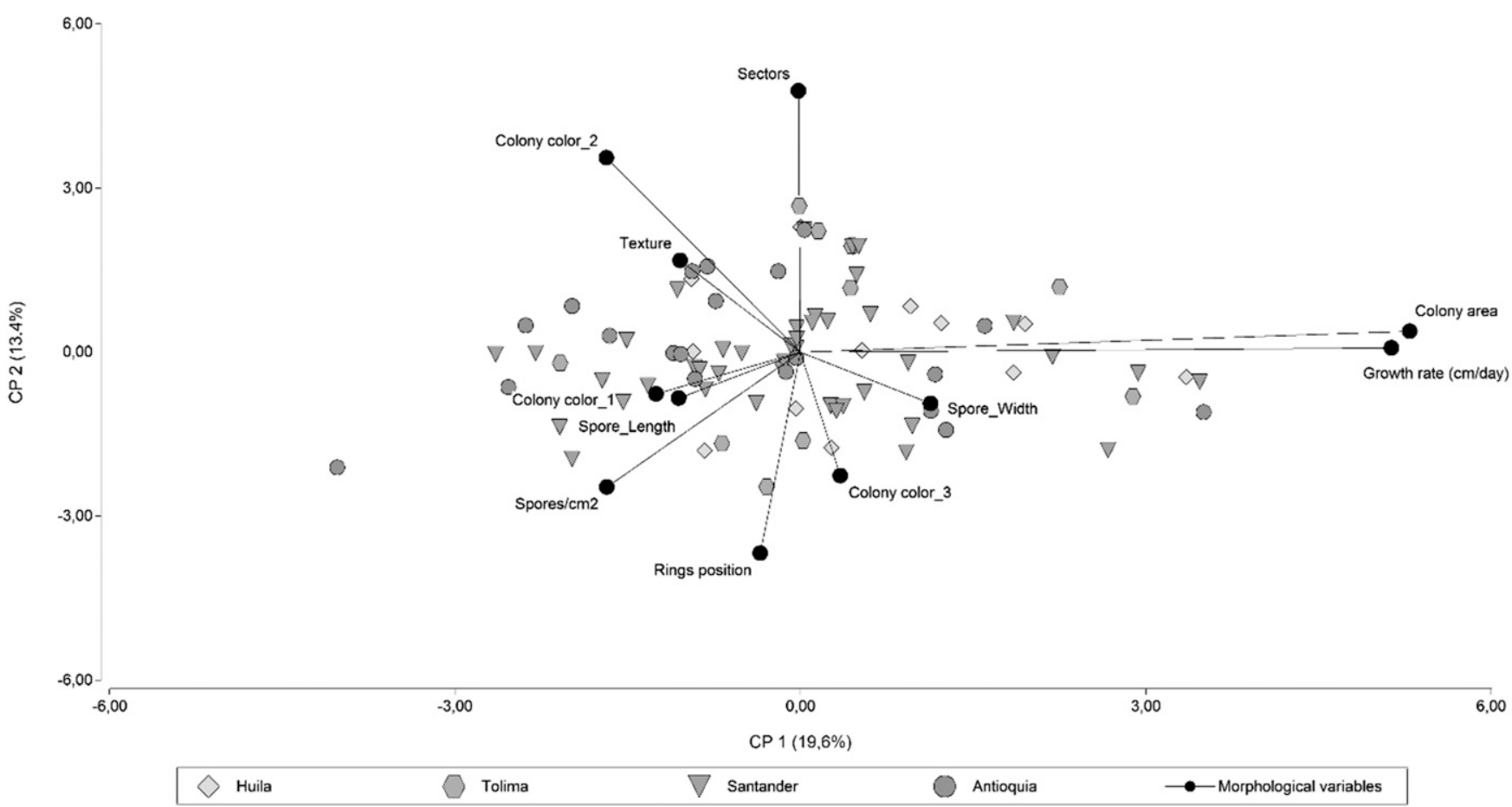

Fig. 2. Biplot of morphological characteristics observed in Moniliophthora roreri isolates based on principal coordinate analysis. First and second components of a principal coordinate analysis of 11 morphological variables. 
by a single isolate. The total gene diversity across all populations was 0.488 (Table 1). Considering a department stratum, the isolate from Santander had the highest gene diversity (0.457; Table 1$)$, while the Huila Department had the lowest (0.277; Table 1).
For the uncorrected and corrected clone populations, the hypothesis of no linkage among markers was rejected $(P<0.001)$ for almost all populations (Fig. 3), indicating a clonal population. The $\check{r} d$ of uncorrected or corrected populations had a little variation from 0.067 to

Table 1. Summary statistics of genetic variation within populations of Moniliophthora roreri isolates according to four strata

\begin{tabular}{|c|c|c|c|c|c|c|c|c|c|c|c|}
\hline Population & $N$ & MLG $^{\mathbf{a}}$ & $\mathbf{e M L G}^{\mathbf{b}}$ & $\mathbf{S E}^{\mathrm{c}}$ & $\mathbf{H}^{\mathbf{d}}$ & $\mathbf{G}^{\mathbf{e}}$ & $\lambda$ & $E_{5}^{\mathrm{f}}$ & Hexpg & $I_{A}{ }^{\mathrm{h}}$ & $\check{r} d^{i}$ \\
\hline \multicolumn{12}{|l|}{ Clone } \\
\hline ICS1 & 18 & 17 & 14.3 & $4.64 \mathrm{e}-01$ & 2.81 & 16.2 & 0.938 & 0.970 & 0.457 & 3.067 & 0.1783 \\
\hline ICS39 & 34 & 34 & 15.0 & $1.61 \mathrm{e}-06$ & 3.53 & 34.0 & 0.971 & 1.000 & 0.451 & 1.573 & 0.0842 \\
\hline ICS95 & 53 & 52 & 14.9 & $2.65 \mathrm{e}-01$ & 3.94 & 51.1 & 0.980 & 0.989 & 0.467 & 0.911 & 0.0513 \\
\hline ICS60 & 15 & 15 & 15.0 & $0.00 \mathrm{e}+00$ & 2.71 & 15.0 & 0.933 & 1.000 & 0.484 & 1.781 & 0.0899 \\
\hline Total & 120 & 117 & 14.9 & $2.41 \mathrm{e}-01$ & 4.75 & 112.5 & 0.991 & 0.975 & 0.488 & 1.284 & 0.0670 \\
\hline \multicolumn{12}{|l|}{ Locality } \\
\hline Rionegro & 10 & 10 & 10.00 & 0.000 & 2.30 & 10.00 & 0.900 & 1.000 & 0.318 & 2.343 & 0.1594 \\
\hline San Vicente & 5 & 5 & 5.00 & 0.000 & 1.61 & 5.00 & 0.800 & 1.000 & 0.395 & -0.404 & -0.0315 \\
\hline Ataco & 7 & 7 & 7.00 & 0.000 & 1.95 & 7.00 & 0.857 & 1.000 & 0.303 & 0.490 & 0.0379 \\
\hline Campo Alegre & 12 & 12 & 10.00 & $\mathrm{NaN}$ & 2.48 & 12.00 & 0.917 & 1.000 & 0.181 & 1.057 & 0.0768 \\
\hline Lebrija & 21 & 21 & 10.00 & $\mathrm{NaN}$ & 3.04 & 21.00 & 0.952 & 1.000 & 0.449 & 2.067 & 0.1164 \\
\hline El Carmen & 15 & 13 & 8.98 & 0.711 & 2.49 & 10.71 & 0.907 & 0.880 & 0.311 & 1.822 & 0.1204 \\
\hline Maceo & 25 & 25 & 10.00 & $\mathrm{NaN}$ & 3.22 & 25.00 & 0.960 & 1.000 & 0.355 & 2.441 & 0.1329 \\
\hline El Playon & 5 & 5 & 5.00 & 0.000 & 1.61 & 5.00 & 0.800 & 1.000 & 0.371 & 5.368 & 0.3838 \\
\hline Apartado & 4 & 4 & 4.00 & 0.000 & 1.39 & 4.00 & 0.750 & 1.000 & 0.254 & 2.127 & 0.3061 \\
\hline Turbo & 1 & 1 & 1.00 & 0.000 & 0.00 & 1.00 & 0.000 & $\mathrm{NaN}$ & $\mathrm{NaN}$ & NA & NA \\
\hline Cimitarra & 3 & 3 & 3.00 & 0.000 & 1.10 & 3.00 & 0.667 & 1.000 & 0.333 & 1.625 & 0.2321 \\
\hline Planadas & 7 & 6 & 6.00 & 0.000 & 1.75 & 5.44 & 0.816 & 0.937 & 0.251 & 2.438 & 0.2474 \\
\hline Rivera & 5 & 5 & 5.00 & 0.000 & 1.61 & 5.00 & 0.800 & 1.000 & 0.359 & 0.153 & 0.0171 \\
\hline Total & 120 & 117 & 9.98 & 0.159 & 4.75 & 112.50 & 0.991 & 0.975 & 0.488 & 1.284 & 0.0670 \\
\hline \multicolumn{12}{|l|}{ Department } \\
\hline Santander & 59 & 57 & 12.9 & $3.59 \mathrm{e}-01$ & 4.02 & 53.6 & 0.981 & 0.959 & 0.457 & 1.716 & 0.0894 \\
\hline Tolima & 13 & 13 & 13.0 & $0.00 \mathrm{e}+00$ & 2.56 & 13.0 & 0.923 & 1.000 & 0.316 & 0.938 & 0.0621 \\
\hline Huila & 18 & 18 & 13.0 & $2.79 \mathrm{e}-07$ & 2.89 & 18.0 & 0.944 & 1.000 & 0.277 & 0.923 & 0.0577 \\
\hline Antioquia & 30 & 30 & 13.0 & $\mathrm{NaN}$ & 3.40 & 30.0 & 0.967 & 1.000 & 0.413 & 2.550 & 0.1360 \\
\hline Total & 120 & 117 & 13.0 & $2.08 \mathrm{e}-01$ & 4.75 & 112.5 & 0.991 & 0.975 & 0.488 & 1.284 & 0.0670 \\
\hline \multicolumn{12}{|l|}{ Altitude } \\
\hline A3 $(400-600 m)$ & 18 & 17 & 9.71 & $4.56 \mathrm{e}-01$ & 2.81 & 16.2 & 0.938 & 0.970 & 0.372 & 1.65 & 0.1013 \\
\hline A5 $(800-1,000 \mathrm{~m})$ & 27 & 26 & 9.87 & $3.34 \mathrm{e}-01$ & 3.24 & 25.1 & 0.960 & 0.979 & 0.465 & 1.27 & 0.0695 \\
\hline A6 $(1,000-1,200 m)$ & 28 & 28 & 10.00 & $3.41 \mathrm{e}-07$ & 3.33 & 28.0 & 0.964 & 1.000 & 0.497 & 1.54 & 0.0873 \\
\hline A4 $(600-800 m)$ & 25 & 25 & 10.00 & $\mathrm{NaN}$ & 3.22 & 25.0 & 0.960 & 1.000 & 0.399 & 2.61 & 0.1495 \\
\hline A2 $(200-400 m)$ & 17 & 17 & 10.00 & $2.31 \mathrm{e}-07$ & 2.83 & 17.0 & 0.941 & 1.000 & 0.284 & 2.25 & 0.1216 \\
\hline $\mathrm{A} 1(0-200 \mathrm{~m})$ & 5 & 5 & 5.00 & $0.00 \mathrm{e}+00$ & 1.61 & 5.0 & 0.800 & 1.000 & 0.208 & 1.30 & 0.1866 \\
\hline Total & 120 & 117 & 9.98 & $1.59 \mathrm{e}-01$ & 4.75 & 112.5 & 0.991 & 0.975 & 0.488 & 1.28 & 0.0670 \\
\hline
\end{tabular}

${ }^{a}$ Multilocus genotypes.

b Number of expected MLG at the smallest sample size $\geq 10$ based on rarefaction.

c Standard error based on eMLG.

d Shannon-Wiener index of MLG diversity.

e Stoddart and Taylor's index of MLG diversity.

${ }^{\mathrm{f}}$ Evenness.

g Nei's 1978 genotypic diversity (corrected for sample size), or expected heterozygosity.

${ }^{\mathrm{h}}$ Index of association.

i Standardized index of association.
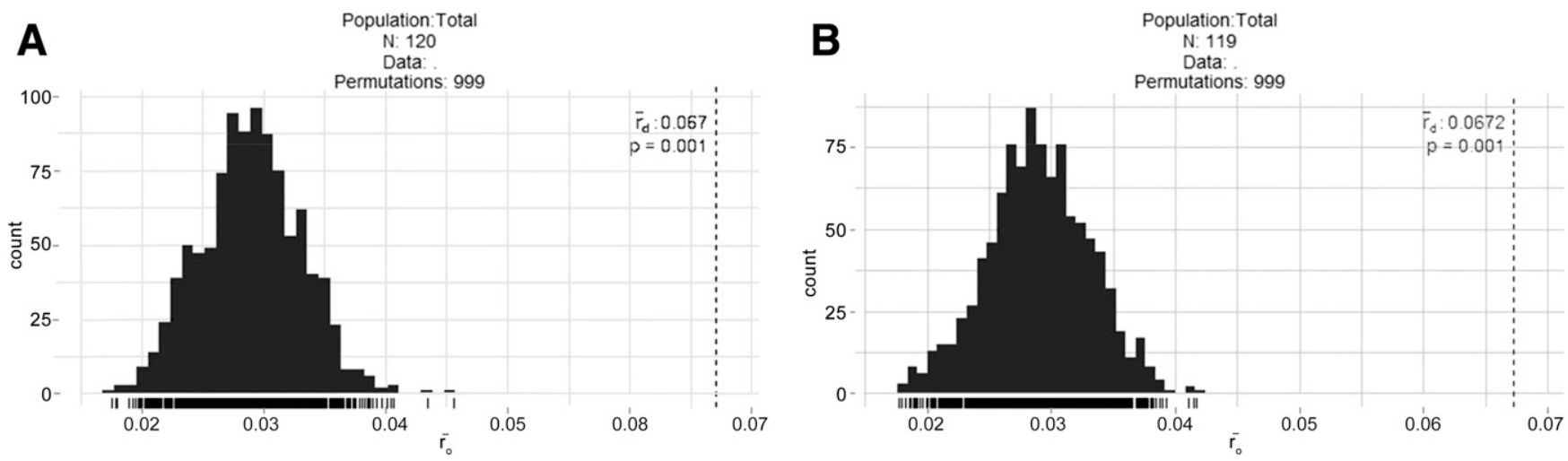

Fig. 3. Index of association standardized to A, uncorrected and B, corrected data, using the poppr package for R Software v1.0.3. 
0.0672 , respectively. Multilocus LD $\left(I_{A}\right.$ and $\check{r} d$ ) was significantly greater than zero within each population $(P<0.001)$. The isolates from Antioquia Department had the highest $I_{A}(2.550$; Table 1) and $\check{r} d\left(0.136\right.$; Table 1), while the Huila Department had the lowest $I_{A}$ (0.923; Table 1) and $\check{r} d(0.057$; Table 1$)$ at the level of department stratum. Given the strong support for linkage in our samples, we interpret the differential heterozygosity between populations as indicative of significant differences in effective population sizes.

Population structure analysis. The Bayesian statistic-based algorithm implemented in STRUCTURE assigned the 120 strains into two (under nonadmixture) or three (under admixture) distinct genetic groups according to Evanno et al. (2005) (Fig. 4). For both analyses, the distribution of genetic groups exhibited high correlation with the geographical location where isolates were collected. When a model nonadmixture was fitted to the data, we found that Santander isolates presented a majority of ancestry from cluster 2 (64.29\% total ancestry from cluster 2 to all individuals in Santander) and a lower contribution of cluster 1 to their ancestry (35.72\%); Antioquia, Tolima, and Huila isolates presented a major component of ancestry from cluster
$1(69.97,93.76$, and $99.9 \%$, respectively) and a progressively smaller contribution of ancestry from cluster $2(30.03,6.24$, and $0.1 \%$, respectively) (Fig. 4B).

Assuming the admixture model for M. roreri populations (Fig. 4A), Santander isolates seemed to conform to a heterogeneous collection, with most individuals having a majority of ancestry from cluster 3 (61.91\% total ancestry of cluster 3 for all individuals in Santander). The second most frequent ancestry in individuals from Santander originated from cluster 1 (31.84\% total ancestry across individuals from Santander) and a minor contribution of ancestry from cluster 2 (6.24\%). On the other hand, isolates from Antioquia had a large ancestry contribution from cluster $1(50.77 \%)$, the second largest contribution originating from cluster $3(34.61 \%)$, and a lesser contribution from cluster $2(14.61 \%)$. Finally, Tolima and Huila isolates presented a major component of ancestry from cluster 2 (85.16 and $90.03 \%$, respectively) and lesser contributions from cluster 3 (12.51 and 5.05\%, respectively) and cluster 1 (2.31 and 4.93\%, respectively) (Fig. 4A).

A comparison of genetic variation within and between two levels of the population hierarchy (department as population) and each one

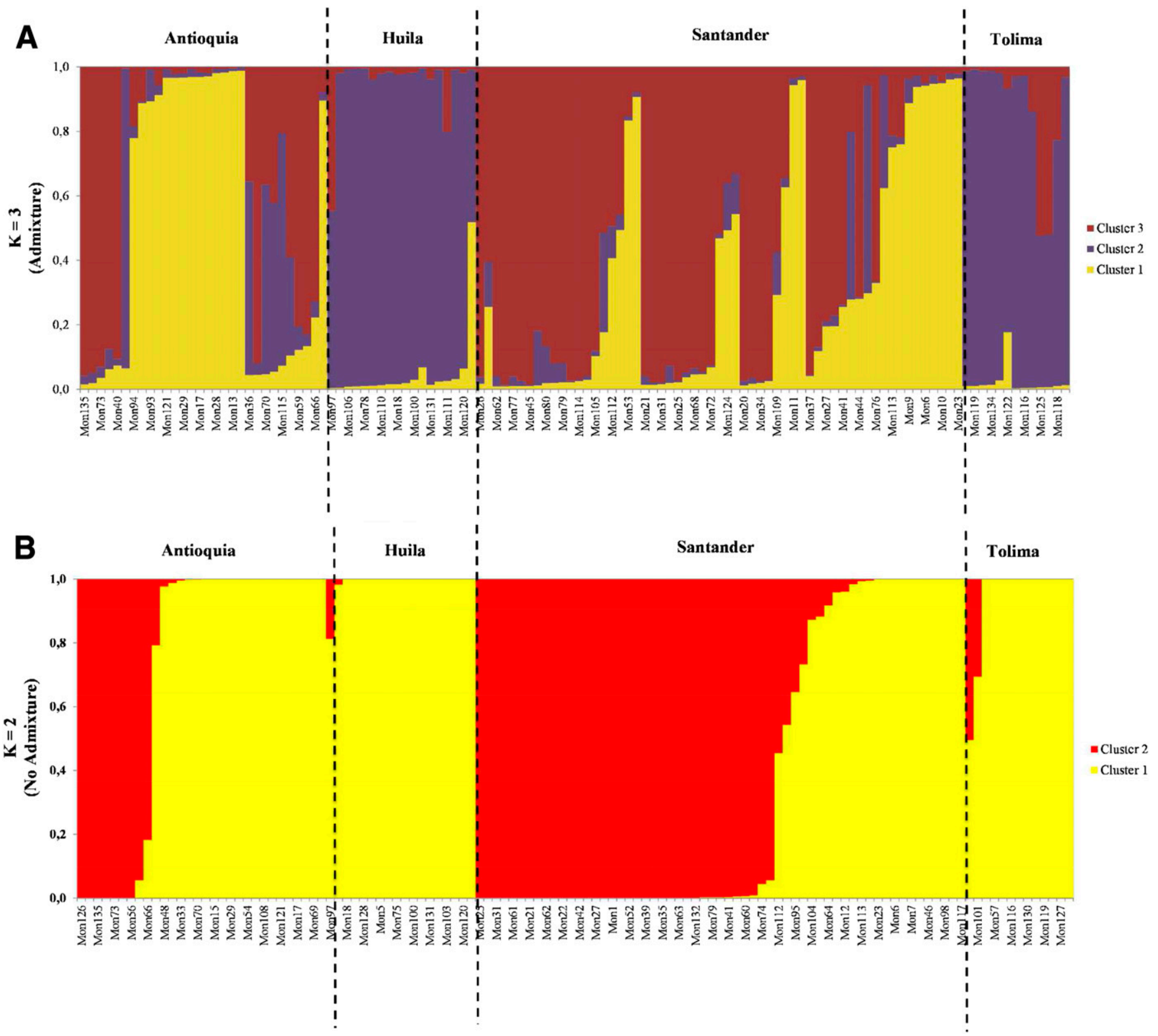

Fig. 4. STRUCTURE analysis of 23 simple-sequence repeat markers for Moniliophthora roreri isolates. Plot of results for STRUCTURE output at different $K$ organized by $Q$ value. Each color represents one cluster defined by STRUCTURE. Vertical bars represent isolates, and the length of each colored segment in each vertical bar represents the proportion contributed by ancestral populations. A, Admixture model and B, no-admixture model. 
of the other strata as subpopulation revealed highly significant subpopulation differentiation $(P=0.001)$ and differentiation when clone and altitude were considered as subpopulations for the analysis $(P=$ 0.002 and $P=0.004$, respectively; Table 2 ). Most haplotype diversity (66.11 to $74.93 \%$ ) was found within the subpopulations (clone, locality, or altitude) but an appreciable amount (25.08 to $33.89 \%$ ) still separated populations (departments) or subpopulations within populations (Table 2), depending on the strata selected as subpopulations for the analysis. When localities and altitude were considered as subpopulations, the $\phi$ values were 0.09 and 0.12 , respectively (Table 2) among the strata considered. These reflect a moderate differentiation between departments' populations and are generally the result of moderate gene flow (Balloux and Lugon-Moulin 2002). The $\phi$ value between populations of $M$. roreri considering clone stratum as subpopulation was 0.17 , reflecting a high degree of differentiation that is generally the result of low gene flow (Balloux and LugonMoulin 2002; Bennett and Stone, 2016).

The first and second components of the PCA performed with SSR data accounted for $15.46 \%$ (PC I) and $9.28 \%$ (PC II) of the total variation, respectively. Principal coordinate analysis separated the isolates into major groups according to geographic location where isolates were collected, which was consistent with the genetic group assignments generated by STRUCTURE (Fig. 5). Similar results were obtained with DAPC (Fig. 6). The strong support for linkage among isolates analyzed, combined with the consistent assignment generated under the STRUCTURE and PCA, allow us to propose that the population structure of $M$. roreri in Colombia can be explained by three main groups.

Interestingly, neighbor-joining tree, based on Nei genetic distance measured from microsatellite data, separated all isolates according to previous defined groupings corresponding to geographic location (Fig. 7).

\section{Discussion}

This is the first study to characterize the $M$. roreri populations in Colombia with a special emphasis on the regions where the pathogen originated. Based solely on morphological characteristics, isolates of different geographical origin could not be differentiated. Ali et al. (2015) obtained similar results because isolates from Colombia and Ecuador did not show differences in spore shapes and sizes (Ali et al. 2015). Because spores are the most important part of the $M$. roreri life cycle, they have been widely studied, and it has been suggested that spore composition, compatible solutes, and other protective proteins inside the spore are influenced by the environmental conditions encountered by the fungus during their formation (Ali et al. 2015). Thus, most of the variation in morphological characteristics measured on the

Table 2. Results of the analysis of molecular variance performed with the 120 Moniliophthora roreri isolates showing a high diversity of multilocus genotypes (MLG) ${ }^{\mathrm{a}}$

\begin{tabular}{|c|c|c|c|}
\hline Subpopulation, hierarchical level & Variation (\%) & $\phi$ & $P^{\mathbf{b}}$ \\
\hline \multicolumn{4}{|l|}{ Clone } \\
\hline$\phi_{\mathrm{CT}}$ (within clones) & 74.93 & 0.25 & 0.001 \\
\hline$\phi_{\mathrm{SC}}$ (among clones within departments) & 8.55 & 0.10 & 0.001 \\
\hline$\phi_{\mathrm{ST}}$ (among departments) & 16.53 & 0.17 & 0.002 \\
\hline \multicolumn{4}{|l|}{ Locality } \\
\hline$\phi_{\mathrm{CT}}$ (within localities) & 68.02 & 0.32 & 0.001 \\
\hline$\phi_{\mathrm{SC}}$ (among localities within departments) & 22.49 & 0.25 & 0.001 \\
\hline$\phi_{\mathrm{ST}}$ (among departments) & 9.49 & 0.09 & 0.021 \\
\hline \multicolumn{4}{|l|}{ Altitude } \\
\hline$\phi_{\mathrm{CT}}$ (within altitudes) & 66.11 & 0.34 & 0.001 \\
\hline$\phi_{\mathrm{SC}}$ (among altitudes within departments) & 22.27 & 0.25 & 0.001 \\
\hline$\phi_{\mathrm{ST}}$ (among departments) & 11.62 & 0.12 & 0.004 \\
\hline \multicolumn{4}{|c|}{$\begin{array}{l}\text { a Relative genetic variances explained by each level of the population hierar- } \\
\text { chy are shown, and } \phi \text { statistics are shown as estimates of subpopulation dif- } \\
\text { ferentiation and gene flow. Department stratum was considered as population } \\
\text { and each one of the other strata as subpopulation. } \\
\text { b } P \text { value was obtained for the } \varphi \text { statistic using a randomization test with } 999 \\
\text { permutations. }\end{array}$} \\
\hline
\end{tabular}

spores might not be heritable. As reported by Díaz-Valderrama and Aime (2016), given that the number of nuclei varies among cells of a single hypha, the subsequent conidiogenesis ensures that varying numbers of nuclei are partitioned into conidia. Thus, the sizes of spores produced by each $M$. roreri isolate varied according to the number of nuclei per conidium. Binucleate spores were significantly larger than mononucleate spores. However, the proportion of binucleate and mononucleate spores seemed to be a characteristic of the isolate, and these could vary significantly among isolates (DíazValderrama and Aime 2016).

The 120 isolates of $M$. roreri collected in this study showed a high level of genetic diversity, and almost each isolate was associated with a single haplotype (117 of 120). Phillips-Mora et al. (2007) reported similar results, with a high level of polymorphism estimated with AFLP and ISSR profiles. In a more recent study, Ali et al. (2015) reported absence of heterozygosity among $M$. roreri isolates from five of the genetic groups previously reported (Ali et al. 2015). These results provided evidence of the clonality of this fungus's populations and their asexual mode of reproduction, as had been suggested for the species and recently supported by Díaz-Valderrama and Aime (2016). However, in the Ali et al. (2015) study, isolates from the upper Magdalena Valley of Colombia showed the highest levels of genetic diversity which, according to the authors, indicated this region as the possible center of origin for $M$. roreri.

We found a high diversity at the population (geographically defined) and isolate level in $M$. roreri, possibly indicating high mutation rates or some levels of genetic exchange within and among the different geographic locations. The high levels of heterozygosis found in this study are consistent with the high mutational rate of microsatellite markers (Balloux and Lugon-Moulin 2002; Hamblin et al. 2007). Additionally, the estimated $I_{A}$ and $\check{r} d$ values suggest that $M$. roreri populations are clonal populations. These coincide with the evidence for the absence of sexual recombination, supporting the hypothesis that this fungus is likely an exclusively anamorphic fungus, as originally described by Ciferri and Parodi (1933) and DíazValderrama and Aime (2016). The fact that strata considered as subpopulations are highly differentiated suggests that they may be sexually reproductively isolated due to behavioral differences or genetic incompatibilities (Bennett and Stone 2016). As to the populations of $M$. roreri, the clone and altitude strata contribute to their differentiation, although these do not define the population structure. If a strict clonal reproduction in $M$. roreri is considered, high levels of gene flow together with a large effective population size would be required to account for genotype diversity.

Ali et al. (2015) suggested dispersal of $M$. roreri clones from the upper Magdalena Valley over time and the subsequent localized genetic drift associated with these unique isolates and events. However, given the high haplotype diversity with LD, this study suggests that, in addition to mutation rates in SSR markers and some gene flow, $M$. roreri could be an asexual species possibly undergoing sporadic or partial recombination due to parasexuality. As reported in Alternaria solani, the genotype diversity of some asexual fungus is comparable with that of other pathogenic fungi characterized by frequent sexual reproduction in their lifecycles (Meng et al. 2015). However, parasexual genetic exchange may be limited in nature due to the prevalence of somatic incompatibility mechanisms in fungi (Anderson and Kohn 1995). According to the AMOVA results, another variation source could be gene flow between subpopulations and populations that, although low to moderate in this fungus, can contribute to the high diversity observed. In fungal pathogens, gene flow can occur naturally through the dispersal of reproductive agents such as spores or can be human-mediated through trade of agricultural products or contamination of transportation tools (Meng et al. 2015).

Considering that fungal populations lacking a sexual cycle are still capable of rapid evolution, this rapid change rate can be reflected in virulence (Michelmore and Hulbert 1987). This could explain several observations such as the high virulence of $M$. roreri isolates, the limited resistance or levels of tolerance seen against this fungus among the cacao germplasms, and the observed increased in disease losses in some tolerant clones in Costa Rica (Ali et al. 2015). 
In our study, the 120 isolates of $M$. roreri were collected from different $T$. cacao clones with different genetic backgrounds but located in the same fields. Our results showed that isolates from different clones were genetically similar, and most of this variation was found between clones, which finally contributed to population differentiation. Our results are consistent, with cacao clone and geographic location playing an important role in shaping population structure. Collecting a single isolate versus many isolates from individual cacao clones represented an advantage for our analysis, because multiples isolates may increase the similarity of isolates in individual geographical populations and overshadow environmental effects. The same effect was observed on the population structure of

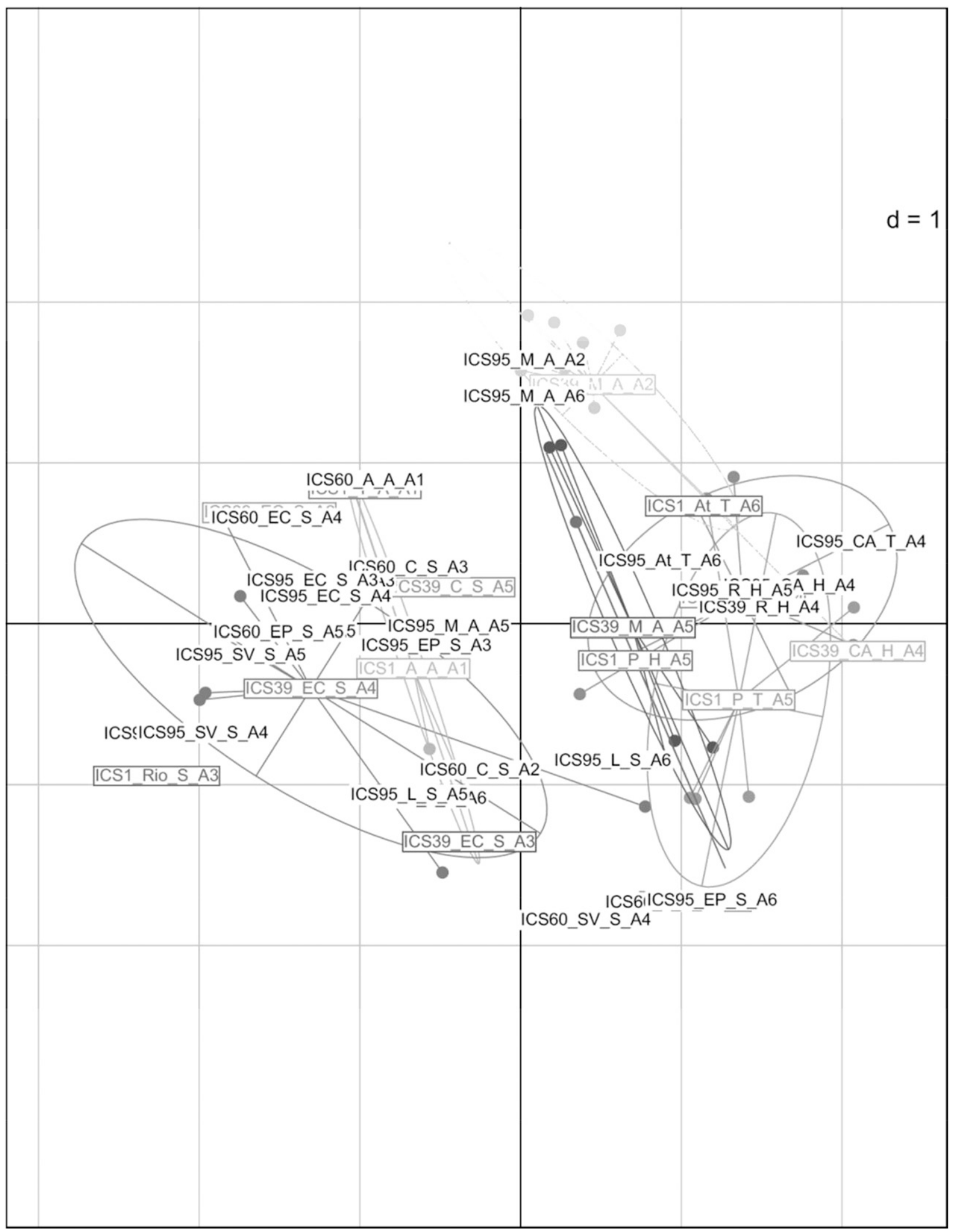

Fig. 5. Scatterplot of the genetic differentiation observed in Moniliophthora roreri isolates based on principal coordinate analysis. First and second component of a principal coordinate analysis for 23 -locus microsatellite genotypes. Groups are shown and inertia ellipses were calculated with $90 \%$ confidence. Dots represent individual strains. The graph scale is given in the upper right corner $(d=0.1)$. 
Villosiclava virens isolates, causal agent of rice false smut, in China. Higher similarity from individual fields but different rice cultivars suggested that the geographic factor played a more important role in the selection of $V$. virens isolates than rice cultivar (Wang et al. 2014).
A genetic analysis of the population structure of $M$. roreri showed three major groups (Fig. 4A). Isolates from Santander presented a large genetic diversity followed by isolates from Antioquia, which presented a large ancestry contribution from cluster 3 and cluster 1 . Isolates from the locations Tolima and Huila presented minor
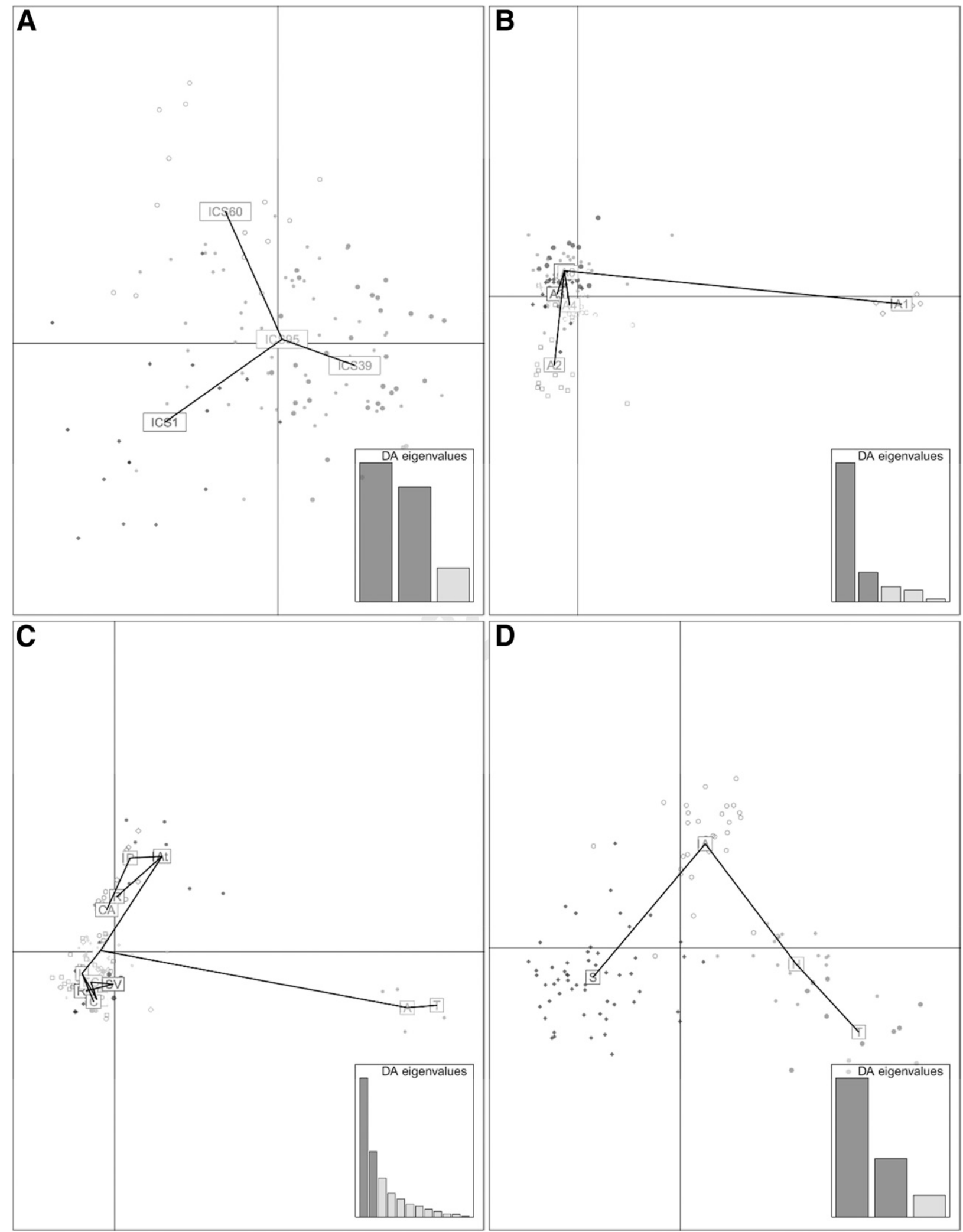

Fig. 6. Summary of genetic relatedness among Moniliophthora roreri obtained from discriminant analysis of principal components (DAPC). Scatter plot of the first two principal components from DAPC with a minimum-spanning tree based on the squared distance between populations, showing the connection between clusters for each stratum. A, Clone stratum; B, altitude stratum; C, locality stratum; and D, department stratum. 
diversity and large ancestry contribution from cluster 2 , with low genetic flow from Santander and Antioquia populations. In our study, the geographical barriers of mountains and the Magdalena River separated the sampling sites. These may have caused the patchy distribution of populations and limited gene flow among some of them. Further analysis using DAPC confirmed the pattern observed on STRUCTURE, with Tolima and Huila isolates separated from Antioquia and Santander isolates (Figs. 4 and 6D). The major Nei genetic distance was observed between Santander and Tolima and Huila populations (Fig. 7), with low levels of gene flow (Fig. 6D).

These results are agreement with Phillips-Mora et al. (2007), who assigned the group names according to origin region as Co-Central, Co-East, and Co-West. It is possible that part of the gene flow we observed between regions has been the consequence of human activities influencing fungus dispersal over long distances, despite the important physical barriers separating some of the areas of cacao production in Colombia (Phillips-Mora et al. 2007). In fact, human-mediated gene flow may account for the higher genetic variation observed in this population, as seen on A. solani (Meng et al. 2015). Those could explain the long-range dispersion of cluster
3 in the Antioquia Department. Isolates collected in Santander showed the largest genetic variability. This variability was reported by PhillipsMora et al. (2007) and Ali et al. (2015), and, in agreement with these authors, our results also suggest that this region could be considered the probable center of origin of the species. Therefore, the Upper Magdalena Valley is considered the most important area in terms of FPR research (Ali et al. 2015).

The results in this study suggest that geographical and environmental differences explain genetic differentiation of $M$. roreri more than the genotype of infected cacao strains. Therefore, control measures such as fungicide applications or selection and deployment of major resistance genes should be implemented with caution, and control measures should attempt to minimize gene flow. Furthermore, disease control strategies should be designed at the regional scale in order to prevent the spread of this fungus from one locality to another.

The role of human activities in disseminating this disease across Latin America and its effects on pathogen population structure support the importance of quarantine politics in order to confine FPR disease to the Latin America area. The same approach has been recommended

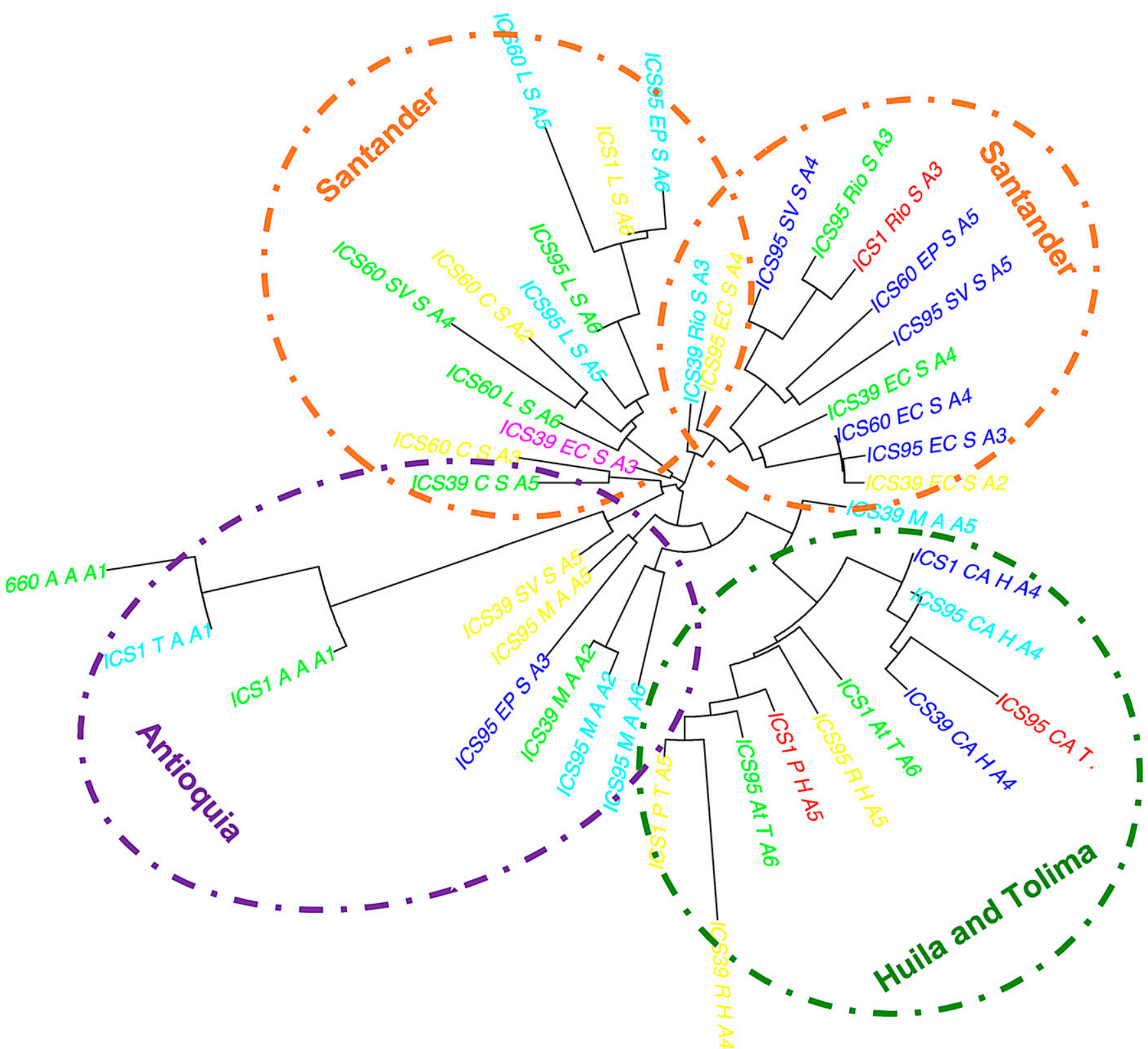

Fig. 7. Dendrogram showing the relationships between Moniliophthora roreri isolates using microsatellite markers. Distances among genotypes were calculated using Nei genetic distance and visualized with an unrooted neighbor-joining tree. Colors represent different geographic locations used in this study. 
for the South American leaf blight disease caused on rubber tree (Hevea brasiliensis) by Pseudocercospora ulei (Barrès et al. 2012). In areas that are still free of the disease, such as Brazil, avoidance is the best control strategy (Phillips-Mora and Wilkinson 2007). Nevertheless, it is important to note that the slow natural spread of FPR to the Brazilian Amazon could be affected by increased human activities in the area (Evans 1986; Phillips-Mora and Wilkinson 2007). Finally, as reported by Phillips-Mora et al. (2007) and Ali et al. (2015) and reported here, the Middle Magdalena Valley of Colombia is considered the most important area in terms of FPR research. The use of pathogen variants from different clusters would ensure the success of breeding and screening programs for new cacao germplasm based on $M$. roreri resistance (Ali et al. 2015) targeting this geographical area.

\section{Acknowledgments}

This research had financial support from the budget assigned to "Corporación Colombiana de Investigación Agropecuaria-CORPOICA" by the Government of Colombia for Science and Technology in the Agricultural Sector.

\section{Literature Cited}

Agapow, P. M., and Burt, A. 2001. Indices of multilocus linkage disequilibrium. Mol. Ecol. Notes 1:101-102.

Ali, S. S., Shao, J., Strem, M. D., Phillips-Mora, W., Zhang, D., Meinhardt, L. W., and Bailey, B. A. 2015. Combination of RNAseq and SNP nanofluidic array reveals the center of genetic diversity of cacao pathogen Moniliophthora roreri in the upper Magdalena Valley of Colombia and its clonality. Front. Microbiol. 6:00850.

Anderson, J. B., and Kohn, L. M. 1995. Clonality in soilborne, plant-pathogenic fungi. Annu. Rev. Phytopathol. 33:369-391.

Arbeláez, L. M. 2010. Análisis de la diversidad intraespecie de Moniliophthora roreri (Cif.) Evans et al. por medio de marcadores morfológicos y genéticos. Magister thesis, Unversidad Nacional de Colombia.

Bailey, B. A., Crozier, J., Sicher, R. C., Strem, M. D., Melnick, R., Carazolle, M. F., Costa, G. G. L., Pereira, G. A. G., Zhang, D., Maximova, S., Guiltinan, M., and Meinhardt, L. 2013. Dynamic changes in pod and fungal physiology associated with the shift from biotrophy to necrotrophy during the infection of Theobroma cacao by Moniliophthora roreri. Physiol. Mol. Plant Pathol. 81:84-96.

Bailey, B. A., Melnick, R. L., Strem, M. D., Crozier, J., Shao, J., Sicher, R., Phillips-Mora, W., Ali, S. S., Zhang, D., and Meinhardt, L. 2014. Differential gene expression by Moniliophthora roreri while overcoming cacao tolerance in the field. Mol. Plant Pathol. 15:711-729.

Balloux, F., and Lugon-Moulin, N. 2002. The estimation of population differentiation with microsatellite markers. Mol. Ecol. 11:155-165.

Barrès, B., Carlier, J., Seguin, M., Fenouillet, C., Cilas, C., and Ravigné, V. 2012. Understanding the recent colonization history of a plant pathogenic fungus using population genetic tools and Approximate Bayesian Computation. Heredity 109:269-279.

Bennett, P., and Stone, J. 2016. Assessments of population structure, diversity, and phylogeography of the Swiss needle cast fungus (Phaeocryptopus gaeumannii) in the U.S. Pacific Northwest. Forests 7:14

Bock, C. H., Wood, B. W., Stevenson, K. L., and Arias, R. S. 2014. Genetic Diversity and Population Structure of Fusicladium effusum on Pecan in the United States. Plant Dis. 98:916-923.

Chao, A., and Shen, T. J. 2003. Nonparametric estimation of Shannon's index of diversity when there are unseen species in sample. Environ. Ecol. Stat. 10:429-443.

Chen, R. S., and McDonald, B. A. 1996. Sexual reproduction plays a major role in the genetic structure of populations of the fungus Mycosphaerella graminicola. Genetics 142:1119-1127.

Ciferri, R., and Parodi, E. 1933. Descrizione del fungo che causa la Moniliasi del cacao. Phytopathol. Z. 6:539-542.

Díaz-Valderrama, J. R., and Aime, M. C. 2016. The cacao pathogen Moniliophthora roreri (Marasmiaceae) produces rhexolytic thallic conidia and their size is influenced by nuclear condition. Mycoscience 57:208-216.

Dutech, C., Enjalbert, J., Fournier, E., Delmontte, F., Barrès, B., Carlier, J., Tharreau, D., and Giraud, T. 2007. Challenges of microsatellite isolation in fungi. Fungal Genet. Biol. 44:933-949.

Earl, D. A., and vonHoldt, B. M. 2012. STRUCTURE HARVESTER: A website and program for visualizing STRUCTURE output and implementing the Evanno method. Conserv. Genet. Resour. 4:359-361.
Evanno, G., Regnaut, S., and Goudet, J. 2005. Detecting the number of clusters of individuals using the software STRUCTURE: A simulation study. Mol. Ecol. 14:2611-2620.

Evans, H. C. 1981. Pod rot of cacao caused by Moniliophthora (Monilia) roreri. Phytopathol. Pap. No. 24. Commonwealth Mycological Institute, London.

Evans, H. C. 1986. A reassessment of Moniliophthora (Monilia) pod rot of cocoa. Cocoa Grow. Bull. 37:34-43

Falush, D., Stephens, M., and Pritchard, J. K. 2003. Inference of population structure using multilocus genotype data: Linked loci and correlated allele frequencies. Genetics 164:1567-1587.

Frenkel, O., Portillo, I., Brewer, M. T., Péros, J. P., Cadle-Davidson, L., and Milgroom, M. G. 2012. Development of microsatellite markers from the transcriptome of Erysiphe necator for analyzing population structure in North America and Europe. Plant Pathol. 61:106-119.

Grünwald, N. J., and Hoheisel, G. A. 2006. Hierarchical analysis of diversity, selfing, and genetic differentiation in populations of the oomycete Aphanomyces euteiches. Phytopathology 96:1134-1141.

Hamblin, M. T., Warburton, M. L., and Buckler, E. S. 2007. Empirical comparison of simple sequence repeats and single nucleotide polymorphisms in assessment of maize diversity and relatedness. PLoS One 2:e1367.

Jia, Q., Gu, Q., Zheng, L., Hsiang, T., Luo, C., and Huang, J. 2015. Genetic analysis of the population structure of the rice false smut fungus, Villosiclava virens, in China using microsatellite markers mined from a genome assembly. Plant Pathol. 64:1440-1449.

Jombart, T. 2008. adegenet: A R package for the multivariate analysis of genetic markers. Bioinformatics 24:1403-1405.

Jombart, T., Devillard, S., and Balloux, F. 2010. Discriminant analysis of principal components: A new method for the analysis of genetically structured populations. BMC Genet. 11:94.

Kamvar, Z. N., Tabima, J. F., and Grünwald, N. J. 2014. Poppr: An R package for genetic analysis of populations with clonal, partially clonal, and/or sexual reproduction. PeerJ 2:e281

Kumar, J., Nelson, R. J., and Zeigler, R. S. 1999. Population structure and dynamic of Magnaporthe grisea in the Indian Himalayas. Genetics 152:971-984.

McDonald, B. A. 1997. The population genetics of fungi: Tools and techniques. Phytopathology 87:448-453.

Meinhardt, L. W., Costa, G. G. L., Thomazella, D. P. T., Teixeira, P. J. P. L., Carazolle, M. F., Schuster, S. C. Carlson, J. E., Guiltinan, M. J., Mieczkowski, P., Farmer, A., Ramaraj, T., Crozier, J., Davis, R. E., Shao, J., Melnick, R., Pereira, G. A. G., and Bailey, B. A. 2014. Genome and secretome analysis of the hemibiotrophic fungal pathogen, Moniliophthora roreri, which causes frosty pod rot disease of cacao: Mechanisms of the biotrophic and necrotrophic phases. BMC Genomics 15:164.

Meng, J. W., Zhu, W., He, M. H., Wu, E. J., Yang, L. N., Shang, L. P., and Zhan, J. 2015. High genotype diversity and lack of isolation by distance in the Alternaria solani populations from China. Plant Pathol. 64:434-441.

Michalakis, Y., and Excoffier, L. 1996. A generic estimation of population subdivision using distances between alleles with special reference for microsatellite loci. Genetics 142:1061-1064.

Michelmore, R. W., and Hulbert, S. H. 1987. Molecular markers for genetic analysis of phytopathogenic fungi. Annu. Rev. Phytopathol. 25:383-404.

Nei, M. 1987. Molecular Evolutionary Genetics. Columbia University Press, New York.

Phillips-Mora, W., Aime, M. C., and Wilkinson, M. J. 2007. Biodiversity and biogeography of the cacao (Theobroma cacao) pathogen Moniliophthora roreri in tropical America. Plant Pathol. 56:911-922.

Phillips-Mora, W., and Wilkinson, M. J. 2007. Frosty pod of cacao: A disease with a limited geographic range but unlimited potential for damage. Phytopathology 97:1644-1647.

Pritchard, J. K., Stephens, M., and Donnelly, P. 2000. Inference of population structure using multilocus genotype data. Genetics 155:945-959.

Stoddart, J. A., and Taylor, J. F. 1988. Genotypic diversity: Estimation and prediction in samples. Genetics 118:705-711.

Thrall, P. H., Burdon, J. J., and Bever, J. D. 2002. Local adaptation in the Linum marginale-Melampsora lini host-pathogen interaction. Evolution 56:1340-1351.

Wang, F., Zhang, S., Liu, M. G., Lin, X. S., Liu, H. J., Peng, Y. L., Lin, Y., Huang, J. B., and Luo, C. X. 2014. Genetic diversity analysis revels that geographic environment plays a more important role than rice cultivar in Villosiclava virens population selection. Appl. Environ. Microbiol. 80:2811-2820.

Wang, X., Lu, P., and Luo, Z. 2013. GMATo: A novel tool for the identification and analysis of microsatellites in large genomes. Bioinformation 9:541-544. 